\title{
Some Results of Intuitionistic Fuzzy Soft Matrix
}

\author{
Mamoni Dhar \\ Department of Mathematics,Science College, Kokrajhar \\ E-mail: mamonidhar@rediffmail.com
}

\begin{abstract}
The purpose of this article is to consider the notions of intuitionistic fuzzy soft matrices and some basic results. This work deals particularly with the definition of transpose of intuitionistic fuzzy soft matrices and then some properties of transpose of intuitionistic fuzzy soft matrices are studied. After that symmetric intuitionistic fuzzy matrices are also defined and some properties are discussed. Numerical examples are provided to make the concept clear.
\end{abstract}

Index Terms-Fuzzy sets, intuitionistic fuzzy sets, intuitionistic fuzzy soft matrices.

\section{INTRODUCTION}

The theory of fuzzy sets introduced by Zadeh[1], showed meaningful applications in many field of studies. This idea of fuzzy sets is welcome because it handles uncertainty and vagueness which the Cantorian set cannot deal with. Fuzzy set has membership function which assigns to each element of the Universe of discourse, a number from the unit interval $[0,1]$, to indicate the degree of belongingness of the set under consideration. In the fuzzy set theory, the membership of an element to a fuzzy set is a single value between zero and one. But in reality, it may not always be true that the degree of non membership of an element in a fuzzy set is equal to one minus the me mbership degree because there may be some hesitation degree as well. Therefore, a generalization of fuzzy set was realized by [2], as intuitionistic fuzzy set which incorporated the degree of hesitation known as the hesitation marg in (and is defined as 1 minus the sum of membership and non membership degrees respectively)

Intuitionistic fuzzy sets as generalization of fuzzy sets can be useful in situation when the description of a problem by linguistic variable, given in terms of membership function only seems to be too difficult to handle. For example, in decision making problem particularly in the case of medical diagnosis, sales analysis, new product marketing, financial services etc, there is a fair chance of a non null hesitation part in each moment of evaluation of an unknown project. To be more precise, intuitionistic fuzzy sets, let us express for example, the fact that the temperature of a patient changes and other symptoms are not quite clear.

In real life situations, most of the problems in economics, social sciences, environment etc, have various uncertainties. However, most of the existing mathematical tools for formal modeling, reasoning and computation are crisp deterministic and precise in character. There are theories namely, theory of probability, evidence, fuzzy set, intuitionistic fuzzy sets, rough sets etc for dealing with uncertainties.

Szmidt and Kacprzyk[3], showed that intuitionistic fuzzy sets are pretty useful in situations when description of a problem by linguistic variable given in terms of membership function only seems too rough. Szmidt and Kacprzyk[4], describes that due to the flexibility of IFS in handling uncertainty, they are tool for a morehuman consistent reasoning under imperfectly defined facts and imprecise knowledge. Atanassov ([5], [6]) carried out rigorous research based on the theory and applications of intuitionistic fuzzy sets.

These theories have their own difficulties as pointed out by Molodtsov[7], and as such the novel concept of soft set theory was initiated. Soft set theory has rich potential for application in solving practical problems in economics, social science, medical science etc. Maji .et.al [8], have studied the theory of fuzzy soft set. Maji. et. al [9], have extended the theory of fuzzy soft set to intuitionistic fuzzy soft sets.

Matrices play a very important role in the broad area of science and technology. However, the classical matrix theory fails to solve the problems involving uncertainties. So there was the need of expressing those uncertainties with fuzzy matrices. Yang and Ji [10], did lot of research on the fuzzy soft matrices and its application in decision making problems.

Broumi. et. al [11], have studied intuitionistic fuzzy soft sets where some some new operations were introduced while defining concentration, dialation and normalization of intuitionistic fuzzy soft sets. Chetia.et.al [12], discussed some results on intuitionistic fuzzy soft matrix theory. Verma. et. al[13], ihas introduced some new results on intuitionistic fuzzy sets by defing some operations/compositions on it. Rajarajeswari. et. al[14], has found some interesting results while dealing with intuitionistic fuzzy soft matrix theory and in applications. Some aspect of these properties can be had from, Dhar [15].

Here in this article, intuitionistic fuzzy soft matrices will be considered and some properties associated with this will be studied.

The paper is organized as follows: Section I is introductory in nature. Section Ii deals with some preliminary concepts which are required in due course of this paper.Section III deals with the properties of transpose of intuitionistic fuzzy soft matrices. Section IV deals with symmetric intuitionistic fuzzy soft matrices and in Section V, the conclusion is provided. 


\section{DENITIONS AND PRELIMINARIES}

A. The basic definitions of intuitionistic fuzzy set theory that are useful in subsequent sections of this article are discussed here.

\subsection{Definition : Soft set}

Suppose that $\mathrm{U}$ is an initial universe $\mathrm{P}(\mathrm{U})$ denote the power set of $U, E$ be the set of all parameters and $A \subseteq E$. A pair $\left(f_{A}, E\right)$ is called a soft set over $\mathrm{U}$ and this is defined by the set of ordered pairs $\left(f_{A}, E\right)=\left\{\left(e, f_{A}(e)\right): e \in E, f_{A}(e) \in P(U)\right\}$ where $f_{A}$ is a mapping given by $f_{A}: E \rightarrow P(U)$. Clearly soft set is a mapping from parameters to $\mathrm{P}(\mathrm{U})$.

\subsection{Definition :Fuzzy soft set}

Suppose that $\mathrm{U}$ is an initial universe of discourse and $\mathrm{E}$ is the set of parameters which involves imprecise words. Let $\mathrm{P}(\mathrm{U})$ denote the power set of $\mathrm{U}$ and $A \subseteq E$. A pair $\left(f_{A}, E\right)$ is called fuzzy soft set over $\mathrm{U}$ where $f_{A}$ is a mapping given by $f_{A}: E \rightarrow P(U)$.

\subsection{Definition: Intuitionistic Fuzzy soft set}

Suppose that $\mathrm{U}$ is an initial universe of discourse and $\mathrm{E}$ is the set of parameters. Let $\mathrm{P}(\mathrm{U})$ denotes the collection of all intuitionistic fuzzy subsets of $\mathrm{U}$. Let $A \subseteq E$. A pair $\left(f_{\mathrm{A}}, E\right)$ is called an intuitionistic fuzzy soft set (IFSS) over $\mathrm{U}$ where $f_{A}$ is a mapping given by $f_{A}: E \rightarrow P(U)$.

\subsection{Definition: Intuitionistic Fuzzy soft Matrix}

Let $\mathrm{U}$ be an initial universe and $\mathrm{E}$ be the set of parameters and $A \subseteq E$.Let $\left(f_{A}, e\right)$ be an intuitionistic fuzzy soft set over $\mathrm{U}$. Then a subset of $U \times E$ is uniquely defined by $R_{A}=\left\{(u, e): e \in A, u \in f_{A}(e)\right\}$, which is called relation form of $\left(f_{A}, e\right)$.The membership and non membership functions are defined by $\mu_{R_{A}}: U \times E \rightarrow[0,1]$ and $v_{R_{A}}: U \times E \rightarrow[0,1]$ where $\mu_{R_{A}}:(u, e) \in[0,1]$ and $v_{R_{A}}:(u, e) \in[0,1]$ are membership value and non membership value of $u \in U$ and $e \in E$.

If $\left(\mu_{i j}, v_{i j}\right)=\left(\mu_{R_{A}}\left(u_{i}, e_{j}\right), v_{R_{A}}\left(u_{i} e_{j}\right)\right)$, we define a matrix

$$
\left[\left(\mu_{i j}, v_{i j}\right)\right]_{m \times n}=\left[\begin{array}{cccc}
\left(\mu_{11}, v_{11}\right) & \left(\mu_{12}, v_{12}\right) & \ldots & \left(\mu_{1 n}, v_{1 n}\right) \\
\left(\mu_{21}, v_{21}\right) & \left(\mu_{22}, v_{22}\right) & \ldots & \left(\mu_{2 n}, v_{2 n}\right) \\
\ldots & \ldots & \ldots & \ldots \\
\left(\mu_{m 1}, v_{m 1}\right) & \left(\mu_{m 2}, v_{m 2}\right) & \ldots & \left(\mu_{m n}, v_{m n}\right)
\end{array}\right]
$$

which is called an $m \times n$ intuitionistic fuzzy soft matrix (IFSM)of the intuitionistic fuzzy soft set $\left(f_{A}, e\right)$ over $\mathrm{U}$.
Therefore, we can say that the intuitionistic fuzzy soft set $\left(f_{A}, e\right)$ over $\mathrm{U}$ is uniquely characterized by the matrix $\left[\left(\mu_{i j}, v_{i j}\right)\right]_{m \times n}$.

Example: Let $U=\left\{u_{1}, u_{2}, u_{3}, u_{4}\right\}$ is a universal set and $E=\left\{e_{1}, e_{2}, e_{3}, e_{4}\right\}$ is a set of parameters. If $A=\left\{e_{1}, e_{2}, e_{3}\right\} \subseteq E$ and

$$
\begin{aligned}
& f_{A}\left(e_{1}\right)=\left\{\left(u_{1}, .4, .5\right),\left(u_{2}, .3, .5\right),\left(u_{3}, .6, .2\right),\left(u_{4}, .5, .2\right)\right\} \\
& f_{A}\left(e_{2}\right)=\left\{\left(u_{1}, .2, .5\right),\left(u_{2}, .3, .4\right),\left(u_{3}, .6, .4\right),\left(u_{4}, .3, .2\right)\right\} \\
& f_{A}\left(e_{3}\right)=\left\{\left(u_{1}, .6, .1\right),\left(u_{2}, .5, .4\right),\left(u_{3}, .3, .4\right),\left(u_{4}, .7, .2\right)\right\}
\end{aligned}
$$

Then the intuitionistic fuzzy set $\left(f_{A}, e\right)$ is a parameterized family $\left\{f_{A}\left(e_{1}\right), f_{A}\left(e_{2}\right), f_{A}\left(e_{3}\right)\right\}$.

Hence the intuitionistic fuzzy soft matrix can be written as

$$
\left(\mu_{i j}, v_{i j}\right)=\left[\begin{array}{llll}
(.4, .5) & (.2, .5) & (.6, .1) & (0,1) \\
(.3, .5) & (.3, .4) & (.5, .4) & (0,1) \\
(.6, .2) & (.6, .4) & (.3, .4) & (0,1) \\
(.5, .2) & (.3, .2) & (.7, .2) & (0,1)
\end{array}\right]
$$

2.5 Definition: Union of Intuitionistic Fuzzy soft Matrices

Let $A=\left[\left(\mu_{i j}^{A}, v_{i j}^{A}\right)\right], B=\left[\left(\mu_{i j}^{B}, v_{i j}^{B}\right)\right] \in I F S M_{m \times n}$. Then union of $\mathrm{A}$ and $\mathrm{B}$ denoted by $A \cup B$ is defined as

$$
A \cup B=\left[\max \left(\mu_{i j}^{A}, \mu_{i j}^{B}\right), \min \left(v_{i j}^{A}, v_{i j}^{B}\right)\right] \text { for all i and j. }
$$

2.6 Definition: Intersection of Intuitionistic Fuzzy soft Matrices

Let $A=\left[\left(\mu_{i j}^{A}, v_{i j}^{A}\right)\right], \quad B=\left[\left(\mu_{i j}^{B}, v_{i j}^{B}\right)\right] \in I F S M_{m \times n}$. Then intersection of $\mathrm{A}$ and $\mathrm{B}$ denoted by $A \cap B$ is defined as

$$
A \cap B=\left[\min \left(\mu_{i j}^{A}, \mu_{i j}^{B}\right), \max \left(v_{i j}^{A}, v_{i j}^{B}\right)\right] \text { for all } \mathrm{i} \text { and } \mathrm{j}
$$

2.7 Definition: Complement of intuitionistic fuzzy soft matrix

Let $A=\left[\left(\mu_{i j}^{A}, v_{i j}^{A}\right)\right] \in I F S M_{m \times n}$ then the complement of A denoted by $A^{o}$ is defined as

$$
A^{o}=\left[\left(v_{i j}^{A}, \mu_{i j}^{A}\right)\right] \text { for all } \mathrm{i} \text { and } \mathrm{j} .
$$


2.8 Definition: Max-min product of intuitionistic fuzzy soft matrices

Let $A=\left[\left(\mu_{i j}^{A}, v_{i j}^{A}\right)\right], B=\left[\left(\mu_{i j}^{B}, v_{i j}^{B}\right)\right] \in \operatorname{IFSM}_{m \times n}$. Then the max-min product of the two intuitionistic fuzzy soft matrices is denoted as $A * B$ is defined as

$$
\begin{aligned}
& A * B=\left[\max -\min \left(\mu_{i j}^{A}, \mu_{i j}^{B}\right), \min -\max \left(v_{i j}^{A}, v_{i j}^{B}\right)\right] \\
& \quad \text { for all i and } \mathrm{j} .
\end{aligned}
$$

2.9 Definition: Scalar multiplication of intuitionistic fuzzy soft matrix

Let $A=\left[\left(\mu_{i j}^{A}, v_{i j}^{A}\right)\right] \in I F S M_{m \times n}$ and k be any scalar. Then the scalar multiplication of intuitionistic fuzzy soft matrix A by the scalar $\mathrm{k}$ is denoted by $\mathrm{kA}$ is defined as

$$
k A=\left[\left(k \mu_{i j}^{A}, k v_{i j}^{A}\right)\right], \text { where } 0 \leq k \leq 1 \text {, for all } \mathrm{i} \text { and } \mathrm{j} .
$$

\subsection{Definition:}

Let

$$
A=\left[\left(\mu_{i j}^{A}, v_{i j}^{A}\right)\right], B=\left[\left(\mu_{i j}^{B}, v_{i j}^{B}\right)\right] \in I F S M_{m \times n}
$$

Then

$A @ B=\left\{<x, \frac{\mu_{i j}{ }^{A}(x)+\mu_{i j}{ }^{B}(x)}{2}, \frac{\gamma_{i j}{ }^{A}(x)+\gamma_{i j}{ }^{B}(x)}{2} \mid x \in X>\right\}$

\subsection{Definition:}

Let

$$
A=\left[\left(\mu_{i j}^{A}, v_{i j}^{A}\right)\right], B=\left[\left(\mu_{i j}^{B}, v_{i j}^{B}\right)\right] \in \operatorname{IFSM}_{m \times n}
$$

Then

$$
A \$ B=\left\{<x, \sqrt{\mu_{i j}{ }^{A}(x) \gamma_{i j}{ }^{B}(x)}, \sqrt{\gamma_{i j}{ }^{A}(x) \gamma_{i j}{ }^{B}(x)} \mid x \in X>\right\}
$$

2.12 Definition: Transpose of intuitionistic fuzzy soft matrix

Let $A=\left[\left(\mu_{i j}^{A}, v_{i j}^{A}\right)\right] \in I F S M_{m \times n}$ then the transpose of A denoted by $A^{T}$ is defined as

$$
A^{T}=\left[\left(\mu_{j i}^{A}, v_{j i}^{A}\right)\right] \text { for all } \mathrm{i} \text { and } \mathrm{j}
$$

The properties of transpose of intuitionistic soft fuzzy matrices will be discussed in the following section.

\section{PROPERT IES OF TRANSPOSE OF INT UITIONIST IC FUZZY SOFT MATRIX}

A. If $A, B$ and $C$ be three intuitionistic fuzzy soft matrices, then the following properties hold

$$
\begin{aligned}
& \text { i. }(A \cup B)^{T}=A^{T} \cup B^{T} \\
& \text { ii. }(A \cap B)^{T}=A^{T} \cap B^{T}
\end{aligned}
$$

iii. $(A B)^{T}=B^{T} A^{T}$

iv. $(k A)^{T}=k A^{T}$

v. $((A \cup B) @ C)^{T}=(A @ C)^{T} \cup(B @ C)^{T}$

vi. $((A \cap B) @ C)^{T}=(A @ C)^{T} \cap(B @ C)^{T}$

\section{Proof of Property $i$ :}

Let $A=\left[\left(\mu_{i j}^{A}, v_{i j}^{A}\right)\right], B=\left[\left(\mu_{i j}^{B}, v_{i j}^{B}\right)\right], C=\left[\left(\mu_{i j}^{C}, v_{i j}^{C}\right)\right]$ be three intuitionistic fuzzy soft matrices. Then

$$
A \cup B=\left[\max \left(\mu_{i j}^{A}, \mu_{i j}^{B}\right), \min \left(v_{i j}^{A}, v_{i j}^{B}\right)\right]
$$

$$
(A \cup B)^{T}=\left[\max \left(\mu_{j i}^{A}, \mu_{j i}^{B}\right), \min \left(v_{j i}^{A}, v_{j i}^{B}\right)\right]
$$

Again

$$
A^{T}=\left(\mu_{j i}^{A}, v_{j i}^{A}\right)
$$

and

$$
B^{T}=\left(\mu_{j i}^{B}, v_{j i}^{B}\right)
$$

$$
A^{T} \cup B^{T}=\left\{\max \left(\mu_{j i}^{A}, \mu_{j i}^{B}\right), \min \left(v_{j i}^{A}, v_{j i}^{B}\right)\right.
$$

Proof of property ii:

$$
\begin{gathered}
A \cap B=\left[\min \left(\mu_{i j}^{A}, \mu_{i j}^{B}\right), \max \left(v_{i j}^{A}, v_{i j}^{B}\right)\right] \\
(A \cap B)^{T}=\left[\min \left(\mu_{i j}^{A}, \mu_{i j}^{B}\right), \max \left(v_{i j}^{A}, v_{i j}^{B}\right)\right] \\
A^{T} \cap B^{T}=\left\{\min \left(\mu_{j i}^{A}, \mu_{j i}^{B}\right), \max \left(v_{j i}^{A}, v_{j i}^{B}\right)\right.
\end{gathered}
$$

Proof of Property iv:

Let

$$
A=\left[\left(\mu_{i j}^{A}, v_{i j}^{A}\right)\right]
$$

Then

$$
k A=\left(k \mu_{i j}^{A}, k v_{i j}^{A}\right)
$$




$$
\begin{gathered}
(k A)^{T}=\left(k \mu_{j i}^{A}, k v_{j i}^{A}\right) \\
k A^{T}=\left(k \mu_{j i}^{A}, k v_{j i}^{A}\right)
\end{gathered}
$$

Hence

$$
(k A)^{T}=k A^{T}
$$

Proof of Property $v$.

Again

$$
(A \cup B) @ C=\left[\left(\mu_{i j}^{A}, v_{i j}^{B}\right)\right] @\left[\left(\mu_{i j}^{C}, v_{i j}^{C}\right)\right]
$$

If

$$
\mu_{i j}^{A} \geq \mu_{i j}^{B} \quad, v_{i j}^{A} \geq v_{i j}^{B}
$$

Then

$$
\begin{gathered}
(A \cup B) @ C=\left[\frac{\mu_{i j}^{A}+\mu_{i j}^{C}}{2}, \frac{v_{i j}^{B}+v_{i j}^{C}}{2}\right] \\
((A \cup B) @ C)^{T}=\left[\frac{\mu_{j i}^{A}+\mu_{j i}^{C}}{2}, \frac{v_{j i}^{B}+v_{j i}^{C}}{2}\right] \\
(A @ C)^{T}=\left[\frac{\mu_{j i}^{A}+\mu_{j i}^{C}}{2}, \frac{v_{j i}^{A}+v_{j i}^{C}}{2}\right] \\
(B @ C)^{T}=\left[\frac{\mu_{j i}^{B}+\mu_{j i}^{C}}{2}, \frac{v_{j i}^{B}+v_{j i}^{C}}{2}\right] \\
\mu_{i j}^{A}+\mu_{i j}^{C} \geq \mu^{B}{ }_{i j}+\mu_{i j}^{C}, v_{i j}^{A}+v_{i j}^{C} \geq v_{i j}^{B}+v_{i j}^{C}, \\
(A @ C)^{T} \cup(B @ C)^{T}=\left[\frac{\mu_{j i}^{A}+\mu_{j i}^{C}}{2}, \frac{v_{j i}^{B}+v_{j i}^{C}}{2}\right]
\end{gathered}
$$

The following numerical example will illustrate the concept clearly.

B. Numerical Examples: Let us consider two fuzzy matrices $A$ and $B$ as follows:

$$
A=\left[\begin{array}{lll}
(.1, .2) & (.5, .4) & (.3, .6) \\
(.5, .4) & (.2, .3) & (.5, .1) \\
(.3, .6) & (.5, .1) & (.6, .2)
\end{array}\right]
$$

and

$$
B=\left[\begin{array}{lll}
(.5, .3) & (.1, .6) & (.7, .1) \\
(.8, .1) & (.4, .3) & (.5, .2) \\
(.2, .5) & (.3, .6) & (.4, .5)
\end{array}\right]
$$

Then we get

$$
\begin{gathered}
A \cup B=\left[\begin{array}{lll}
(.5, .2) & (.5, .4) & (.7, .1) \\
(.8, .1) & (.4, .3) & (.5, .1) \\
(.5, .2) & (.3, .4) & (.6, .2)
\end{array}\right] \\
A \cap B=\left[\begin{array}{lll}
(.1, .3) & (.1, .6) & (.3, .6) \\
(.4, .4) & (.2, .3) & (.5, .2) \\
(.2, .5) & (.3, .6) & (.4, .5)
\end{array}\right] \\
(A \cup B)^{T}=\left[\begin{array}{lll}
(.5, .2) & (.8, .1) & (.5, .2) \\
(.5, .4) & (.4, .3) & (.3, .4) \\
(.7, .1) & (.5, .1) & (.6, .2)
\end{array}\right] \\
(A \cap B)^{T}=\left[\begin{array}{lll}
(.1, .3) & (.1, .6) & (.2, .5) \\
(.1, .6) & (.2, .3) & (.3, .6) \\
(.3, .6) & (.5, .1) & (.4, .5)
\end{array}\right]
\end{gathered}
$$

Again we have

$$
\begin{aligned}
B^{T} & =\left[\begin{array}{lll}
(.5, .3) & (.8, .1) & (.2, .5) \\
(.1, .6) & (.4, .3) & (.3, .6) \\
(.7, .1) & (.5, .2) & (.4, .5)
\end{array}\right] \\
A^{T} & =\left[\begin{array}{lll}
(.1, .2) & (.4, .4) & (.5, .2) \\
(.5, .4) & (.2, .3) & (.3, .4) \\
(.3, .6) & (.5, .1) & (.6, .2)
\end{array}\right]
\end{aligned}
$$

Then

$$
A^{T} \cup B^{T}=\left[\begin{array}{lll}
(.5, .2) & (.8, .1) & (.5, .2) \\
(.5, .4) & (.4, .3) & (.3, .4) \\
(.7, .1) & (.5, .1) & (.6, .2)
\end{array}\right]
$$

and

$$
A^{T} \cap B^{T}=\left[\begin{array}{lll}
(.1, .3) & (.1, .6) & (.2, .5) \\
(.1, .6) & (.2, .3) & (.3, .6) \\
(.3, .6) & (.5, .1) & (.4, .5)
\end{array}\right]
$$

Now we shall calculate the product $A B$ of these two intuitionistic fuzzy matrices in the following way 
$C_{11}=[\max \{\min (1,5), \min (, 5,8), \min (3,2)\}, \min \{\max (2,3), \max (4,1), \max (6,6,5)\}]$ $=\{\max (.1, .5, .2), \min (.3, .4, .6)\}$ $=(.5, .3)$

$C_{12}=[\max \{\min (1,1,1), \min (, 5,4), \min (3,3)\}, \min \{\max (2,6), \max (4,3), \max (6,6)\}]$ $=\{\max (.1, .4, .3), \min (.6, .4, .6)\}$

$=(.4,4)$

$C_{13}=[\max \{\min (1,7), \min (5,5), \min (3,4)\}, \min \{\max (2,1), \max (4,2), \max (6,5)\}]$ $=\{\max (.1, .5, .3), \min (.2, .4, .6)\}$ $=(.5, .2)$

$C_{21}=[\max \{\min (4,5), \min (2,8), \min (, 5,2)\}, \min \{\max (4,3), \max (3,1), \max (1,1,5)\}]$ $=\{\max (.4, .2, .2), \min (.4, .3, .5)\}$

$=(.4, .3)$

$C_{22}=[\max \{\min (4,1), \min (2,4), \min (, 5,3)\}, \min \{\max (4,4), \max (3,3), \max (1,1,6)\}]$ $=\{\max (.1, .2, .3), \min (.6, .3, .6)\}$

$=(.3,3)$

$\left.C_{2 \mathrm{~B}}=[\max \{\min (, 4,7), \min (2,5), \min (, 5,4)\}, \min \{\max (4,1), \max (3,2), \max (1,5))\}\right]$ $=\{\max (.4, .2, .4), \min (.4, .3, .5)\}$ $=(.4,3)$

$C_{71}=[\max \{\min (.5,5), \min (3,8), \min (6,2)\}, \min \{\max (2,3), \max (4,1), \max (2,5)\}]$ $=\{\max (.5, .3, .2), \min (.3, .4, .5)\}$ $=(.5, .3)$

$C_{22}=[\max \{\min (2,5), \min (3,4), \min (4,3)\}, \min \{\max (2,6), \max (4,3), \max (.2,5)\}]$ $=\{\max (.2, .2, .3), \min (.6, .4, .5)\}$ $=(.3,4)$

$C_{2 \mathrm{~B}}=[\max \{\min (.5,7), \min (4,5), \min (4,6)\}, \min \{\max (2,1), \max (4,2), \max (5,5,2)\}]$ $=\{\max (.5, .4, .4), \min (.2, .4, .5)\}$

$=(.5, .2)$

Thus we get

$$
A B=\left[\begin{array}{lll}
(.5, .3) & (.4, .4) & (.5, .2) \\
(.4, .3) & (.3, .3) & (.4, .3) \\
(.5, .3) & (.3, .4) & (.5, .2)
\end{array}\right]
$$

And

$$
(A B)^{T}=\left[\begin{array}{lll}
(.5, .3) & (.4, .3) & (.5, .3) \\
(.4, .4) & (.3, .3) & (.3, .4) \\
(.5, .2) & (.4, .3) & (.5, .2)
\end{array}\right]
$$

Then let us compute $B^{T} A^{T}$ and if $B^{T} A^{T}=\left[D_{i j}\right]$, we get

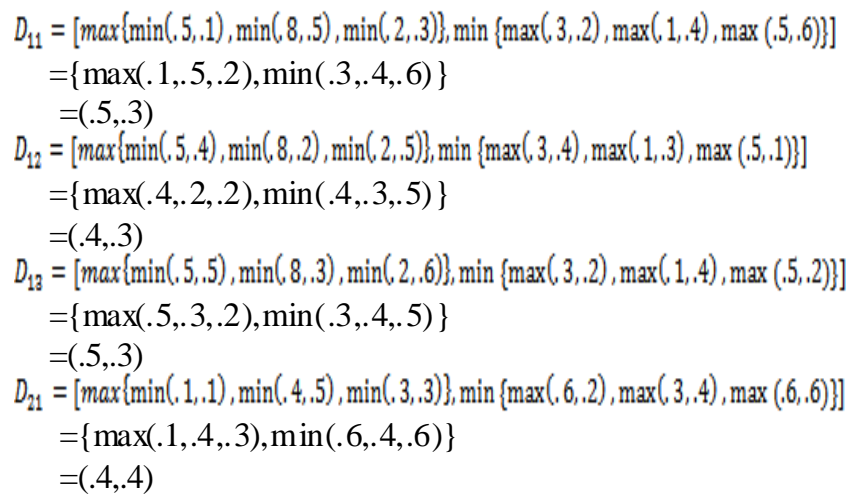

$D_{11}=[\max \{\min (.5,1), \min (8,5), \min (2,3)\}, \min \{\max (3,2), \max (1,4), \max (5,5)\}]$ $=\{\max (.1, .5, .2), \min (.3, .4, .6)\}$

$D_{12}=[\max \{\min (.5,4), \min (8,2), \min (2,5)\}, \min \{\max (3,4), \max (1,3), \max (.5,1)\}]$ $=\{\max (.4, .2, .2), \min (.4, .3, .5)\}$

$=[\max \{\min (.5,5), \min (8,3), \min (2,6)\}, \min \{\max (3,2,2), \max (1,4), \max (.5,2)\}]$ $=\{\max (.5, .3, .2), \min (.3, .4, .5)\}$

$=[\max \{\min (1,1,1), \min (4,5), \min (3,3)\}, \min \{\max (6,6), \max (3,4), \max (6,6)\}]$ $=(.4, .4)$

$D_{22}=[\max \{\min (1,4), \min (4,2), \min (3,5)\}, \min \{\max (6,4), \max (3,3), \max (6,6,1)\}]$ $=\{\max (.1, .2, .3), \min (.6, .3, .6)\}$ $=(.3, .3)$

$D_{28}=[\max \{\min (1,5), \min (4,3), \min (, 3,6)\}, \min \{\max (6,2), \max (3,4), \max (6,6,2)\}]$ $=\{\max (.1, .3, .3), \min (.6, .4, .6)\}$ $=(.3,4)$

$D_{31}=[\max \{\min (7,1), \min (, 5,5), \min (4,3)\}, \min \{\max (1,2), \max (2,4), \max (5,5)\}]$ $=\{\max (.1, .5, .3), \min (.2, .4, .6)\}$ $=(.5, .2)$

$D_{22}=[\max \{\min (7,4), \min (, 5,2), \min (4,5)\}, \min \{\max (1,4), \max (2,3), \max (5,1)\}]$ $=\{\max (.4, .2, .4), \min (.4, .3, .5)\}$ $=(.4,3)$

$D_{\mathrm{ag}}=[\max \{\min (.7,5), \min (.5,3), \min (, 4,6)\}, \min \{\max (1,2), \max (2,4), \max (.5,2)\}]$ $=\{\max (.5, .3, .4), \min (.2, .4, .5)\}$ $=(.5, .2)$

Hence

$$
B^{T} A^{T}=\left[\begin{array}{lll}
(.5, .3) & (.4, .3) & (.5, .3) \\
(.4, .4) & (, 3, .3) & (.3, .4) \\
(.5, .2) & (.4, .3) & (, 3, .1)
\end{array}\right]
$$

Let us chose $\mathrm{k}$ such that $k \in[0,1]$. Then we have

$$
\begin{gathered}
k A=\left[\begin{array}{ccc}
(.05, .1) & (.25, .2) & (.15, .3) \\
(.2, .2) & (.1, .15) & (.25, .05) \\
(.25, .1) & (.15, .2) & (.3, .1)
\end{array}\right] \\
(k A)^{T}=\left[\begin{array}{lll}
(.05, .1) & (.2, .2) & (.25, .1) \\
(.25, .2) & (.1, .15) & (.15, .2) \\
(.15, .3) & (.25, .05) & (.3, .1)
\end{array}\right]
\end{gathered}
$$

and

$$
k A^{T}=\left[\begin{array}{ccc}
(.05, .1) & (.2, .2) & (.25, .1) \\
(.25, .2) & (.1, .15) & (.15, .2) \\
(.15, .3) & (.25, .05) & (.3, .1)
\end{array}\right]
$$

Let us consider three intuitionistic fuzzy soft matrices $\mathrm{A}, \mathrm{B}$ and $\mathrm{C}$ as

$$
\begin{gathered}
A=\left[\begin{array}{lll}
(.1, .2) & (.5, .4) & (.3, .6) \\
(.4, .4) & (.2, .3) & (.5, .2) \\
(.5, .2) & (.3, .4) & (.6, .2) \\
(.7, .2) & (.6, .1) & (.5, .3)
\end{array}\right] \\
B=\left[\begin{array}{lll}
(.5, .3) & (.1, .6) & (.7, .1) \\
(.8, .1) & (.4, .3) & (.5, .2) \\
(.2, .5) & (.3, .6) & (.4, .3) \\
(.1, .7) & (.2, .5) & (.5, .2)
\end{array}\right],
\end{gathered}
$$




$$
C=\left[\begin{array}{lll}
(.1, .3) & (.1, .6) & (.3, .6) \\
(.4, .4) & (.2, .3) & (.5, .2) \\
(.2, .5) & (.3, .6) & (.4, .5) \\
(.1, .7) & (.2, .5) & (.5, .2)
\end{array}\right]
$$

Then

$$
\begin{aligned}
& A \cup B=\left[\begin{array}{lll}
(.5, .2) & (.5, .4) & (.7, .1) \\
(.8, .1) & (.4, .3) & (.5, .1) \\
(.5, .2) & (.3, .4) & (.6, .2) \\
(.7, .2) & (.6, .1) & (.5, .1)
\end{array}\right] \\
&(A \cup B) @ C \\
&=\left[\begin{array}{ccc}
(.3, .25) & (.3, .5) & (.5, .35) \\
(.6, .25) & (.3, .3) & (.5, .15) \\
(.35, .35) & (.3, .5) & (.5, .35) \\
(.4, .45) & (.4, .3) & (.5, .2)
\end{array}\right]
\end{aligned}
$$

$$
\begin{aligned}
& (A @ C)^{T} \cap((B @ C))^{T} \\
& =\left[\begin{array}{cccc}
(.1, .35) & (.4, .45) & (.25, .5) & (.15, .7) \\
(.1, .6) & (.2, .3) & (.3, .6) & (.2, .5) \\
(.5, .6) & (.5, .2) & (.3, .5) & (.5, .3)
\end{array}\right]
\end{aligned}
$$

Hence

$$
\begin{aligned}
& (A @ C)^{T} \cup((B @ C))^{T} \\
& =\left[\begin{array}{cccc}
(.3, .25) & (.6, .23) & (.35, .35) & (.4, .45) \\
(.3, .5) & (.3, .3) & (.3, .5) & (.4, .3) \\
(.5, .35) & (.5, .15) & (.3, .35) & (.5, .2)
\end{array}\right]
\end{aligned}
$$

and

Hence

$$
\begin{aligned}
& ((A \cup B) @ C))^{T} \\
& =\left[\begin{array}{cccc}
(.3, .25) & (.6, .25) & (.35, .35) & (.4, .45) \\
(.3, .5) & (.3, .3) & (.3, .5) & (.4, .3) \\
(.5, .35) & (.5, .15) & (.5, .35) & (.5, .2)
\end{array}\right]
\end{aligned}
$$

IV. SYMMETRIC INTUITIONIST IC FUZZY SOFT MATRIX

Again

$$
A @ C=\left[\begin{array}{ccc}
(.1, .25) & (.3, .5) & (.3, .6) \\
(.4, .4) & (.2, .3) & (.5, .15) \\
(.35, .35) & (.3, .5) & (.5, .35) \\
(.4, .45) & (.4, .3) & (.5, .3)
\end{array}\right]
$$

A. Let $A=\left[\left(\mu_{i j}^{A}, v_{i j}^{A}\right)\right] \in I F S M_{m \times n}$ and if $m=n$, $\mu_{i j}^{A}=\mu_{j i}^{A}$ and $v_{i j}^{A}=v_{j i}^{A}$ then the matrix $A$ is said to be symmetric intuitionistic fuzzy soft matrix. In other words, a matrix $A$ will be said to be a symmetric fuzzy soft matrix if $A^{T}=A$

For example

$$
A=\left(\begin{array}{lll}
(.1, .2) & (.5, .4) & (.5, .2) \\
(.5, .4) & (.2, .3) & (.5, .1) \\
(.5, .2) & (.5, .1) & (.6, .2)
\end{array}\right)
$$

and

$$
B @ C=\left[\begin{array}{ccc}
(.3, .3) & (.1, .6) & (.5, .35) \\
(.6, .25) & (.3, .3) & (.5, .2) \\
(.2, .5) & (.3, .6) & (.4, .5) \\
(.1, .7) & (.2, .5) & (.5, .2)
\end{array}\right]
$$

Then

$$
\begin{aligned}
& ((A @ C))^{T} \\
& =\left[\begin{array}{llll}
(.1, .3) & (.4, .45) & (.4, .35) & (.45, .45) \\
(.3, .5) & (.2, .3) & (.3, .5) & (.4, .3) \\
(.3, .6) & (.5, .2) & (.5, .35) & (.5, .3)
\end{array}\right]
\end{aligned}
$$

is a symmetric intuitionistic fuzzy soft matrix.

\section{B. Propositions on Symmetric intuitionistic fuzzy soft} matrices

i. If $\mathrm{A}$ and $\mathrm{B}$ be two symmetric intuitionistic fuzzy matrix then $A \cup B$ is again a symmetric intuitionistic fuzzy soft matrix.

ii. If $\mathrm{A}$ and $\mathrm{B}$ be two symmetric intuitionistic fuzzy matrix then $A \cap B$ is again a symmetric intuitionistic fuzzy soft matrix.

iii. If $\mathrm{A}$ be a symmetric intuitionistic fuzzy soft matrix then $\mathrm{kA}$ is again a symmetric intuitionistic fuzzy soft matrix.

iv. If $\mathrm{A}$ and $\mathrm{B}$ be two symmetric intuitionistic fuzzy matrix then $A @ B$ is again a symmetric intuitionistic fuzzy soft matrix.

v. If $\mathrm{A}$ and $\mathrm{B}$ be two symmetric intuitionistic fuzzy 
matrix, then $A \$ B$ is again a symmetric intuitionistic fuzzy soft matrix.

vi. If $\mathrm{A}$ is a symmetric intuitionistic fuzzy soft matrix then $\mathrm{A}^{\mathrm{T}} \mathrm{A}$ and $\mathrm{AA}^{\mathrm{T}}$ are also symmetric intuitionistic fuzzy soft matrix.

vii. If $A$ and $B$ are symmetric intuitionistic fuzzy soft matrix then $\mathrm{A}^{\mathrm{T}} \mathrm{BA}$ is again a symmetric intuitionistic fuzzy soft matrix.

viii. If $\mathrm{A}, \mathrm{B}$ and $\mathrm{C}$ be two symmetric intuitionistic fuzzy matrix, then $(A \cup B) \$ C$ is again a symmetric intuitionistic fuzzy soft matrix.

ix. If $\mathrm{A}, \mathrm{B}$ and Cbe two symmetric intuitionistic fuzzy matrix, then $(A \cap B) \$ C$ is again a symmetric intuitionistic fuzzy soft matrix.

$\mathrm{x}$. If $\mathrm{A}, \mathrm{B}$ and $\mathrm{C}$ be two symmetric intuitionistic fuzzy matrix, then $(A @ B) \$ C$ is again a symmetric intuitionistic fuzzy soft matrix.

\section{Proofs of the Propositions with examples}

\section{Proof of $i$}

If $\mathrm{A}$ and $\mathrm{B}$ be two symmetric intuitionistic fuzzy soft matrix then $A^{T}=A$ and $B^{T}=B$. Now we are to show that $A \cup B$ is again a symmetric intuition istic fuzzy soft matrix.

We have

$$
(A \cup B)^{T}=A^{T} \cup B^{T}
$$

Since $\quad A^{T}=A \quad$ and $\quad B^{T}=B \quad, \quad$ then $A^{T} \cup B^{T}=A \cup B \quad$ which implies that $(A \cup B)^{T}=A \cup B$

Thus the result follows that if $\mathrm{A}$ and $\mathrm{B}$ be two symmetric intuitionistic soft fuzzy matrices then $A \cup B$ is again a symmetric intuitionistic fuzzy soft matrix.

\section{Numerical example}

Let $\mathrm{A}, \mathrm{B}$ and $\mathrm{C}$ be three symmetric intuitionistic soft fuzzy matrices such that

$$
\begin{aligned}
A & =\left(\begin{array}{lll}
(.1, .2) & (.5, .4) & (.3, .6) \\
(.5, .4) & (.2, .3) & (.5, .1) \\
(.3, .6) & (.5, .1) & (.6, .2)
\end{array}\right) \\
B & =\left(\begin{array}{lll}
(.5, .4) & (.6, .3) & (.8, .2) \\
(.6, .3) & (.7, .2) & (.4, .6) \\
(.8, .2) & (.4, .6) & (.8, .1)
\end{array}\right)
\end{aligned}
$$

and

$$
C=\left(\begin{array}{lll}
(.3, .4) & (.5, .4) & (.6, .1) \\
(.5, .4) & (.6, .1) & (.4, .6) \\
(.6, .1) & (.4, .6) & (.7, .2)
\end{array}\right)
$$

Proof of $i$

$$
\begin{gathered}
A \cup B=\left(\begin{array}{lll}
(.5, .2) & (.6, .3) & (.8, .2) \\
(.6, .3) & (.7, .2) & (.5, .1) \\
(.8, .2) & (.5, .1) & (.8, .1)
\end{array}\right) \\
(A \cup B)^{T}=\left(\begin{array}{lll}
(.5, .2) & (.6, .3) & (.8, .2) \\
(.6, .3) & (.7, .2) & (.5, .1) \\
(.8, .2) & (.5, .1) & (.8, .1)
\end{array}\right)
\end{gathered}
$$

From which it is clear that

$$
(A \cup B)^{T}=A \cup B
$$

Proof of $i i$

We have

$$
(A \cap B)^{T}=A^{T} \cap B^{T}
$$

Proceeding in the above manner, the required result can be obtained.

\section{Proof of iii}

Let $\mathrm{A}$ be any symmetric intuitionistic fuzzy soft matrix and $\mathrm{k}$ be any scalar, and then it is to be proved that $\mathrm{kA}$ is again a symmetric intuitionistic fuzzy soft matrix.

Given A is a symmetric intuitionistic fuzzy soft matrix, then $\mu_{i j}^{A}=\mu_{j i}^{A}$ and $v_{i j}^{A}=v_{j i}^{A}$

Here it is to be proven that $\mathrm{kA}$ is again a symmetric intuitionistic fuzzy soft matrix. That is to prove that $(k A)^{T}=k A$

Now

$$
\begin{aligned}
k A & =k\left(\mu_{i j}{ }^{A}, v_{i j}{ }^{A}\right) \\
& =\left(k \mu_{i j}{ }^{A}, k v_{i j}{ }^{A}\right)
\end{aligned}
$$

Again

$$
\begin{aligned}
(k A)^{T} & =\left(k \mu_{j i}{ }^{A}, k v_{j i}^{A}\right) \\
& =\left(k \mu_{i j}{ }^{A}, k v_{i j}^{A}\right)
\end{aligned}
$$

It proves $\mathrm{iii}$.

Numerical Example

Let

$$
A=\left(\begin{array}{lll}
(.1, .2) & (.5, .4) & (.3, .6) \\
(.5, .4) & (.2, .3) & (.5, .1) \\
(.3, .6) & (.5, .1) & (.6, .2)
\end{array}\right)
$$

and $\mathrm{k}=.5$ 
Then

$$
k A=\left(\begin{array}{ccc}
(.05, .1) & (.25, .2) & (.15, .30) \\
(.25, .2) & (.1, .15) & (.25, .05) \\
(.15, .30) & (.25, .05) & (.30, .1)
\end{array}\right)
$$

Again

$$
(k A)^{T}=\left(\begin{array}{ccc}
(.05, .1) & (.25, .2) & (.15, .30) \\
(.25, .2) & (.1, .15) & (.25, .05) \\
(.15, .30) & (.25, .05) & (.30, .1)
\end{array}\right)
$$

This shows that

$$
(k A)^{T}=k A
$$

and hence $\mathrm{kA}$ is again a symmetric intuitionistic fuzzy soft matrix.

$$
(k A)^{T}=k A
$$

\section{Proof of iv}

Let $A=\left(\mu_{i j}^{A}, v_{i j}^{A}\right)$ and $B=\left(\mu_{i j}^{B}, v_{i j}^{B}\right)$ be two symmetric intuitionistic fuzzy soft matrices. Then it is to be proved that $(A @ B)^{T}=A @ B$

Here

$$
A @ B=\left(\frac{\mu_{i j}^{A}+\mu_{i j}^{B}}{2}, \frac{v_{i j}^{A}+v_{i j}^{B}}{2}\right)
$$

and

$$
(A @ B)^{T}=\left(\frac{\mu_{j i}^{A}+\mu_{j i}^{B}}{2}, \frac{v_{j i}^{A}+v_{j i}^{B}}{2}\right)
$$

Since A and B are two symmetric intuitionistic fuzzy soft matrices then $\mu_{i j}^{A}=\mu_{j i}^{A}$ and $v_{i j}^{A}=v_{j i}^{A}$

From the above, it is clear that $(A @ B)^{T}=A @ B$

\section{Numerical Example}

If the above two intuitionistic fuzzy soft matrices are considered, then we see that

$$
A @ B=\left(\begin{array}{ccc}
(.3, .3) & (.55, .35) & (.55, .4) \\
(.55, .35) & (.45, .25) & (.45, .35) \\
(.55, .4) & (.45, .35) & (.65, .2))
\end{array}\right)
$$

$$
(A @ B)^{T}=\left(\begin{array}{ccc}
(.3, .3) & (.55, .35) & (.55, .4) \\
(.55, .35) & (.45, .25) & (.45, .35) \\
(.55, .4) & (.45, .35) & (.65, .2))
\end{array}\right)
$$

and the above result shows that intuitionistic fuzzy soft matrix $A @ B$ is again a symmetric intuitionistic fuzzy soft matrix.

\section{Proof ofv}

If the above two intuitionistic fuzzy soft matrices are considered, then we see that

$$
A \$ B=\left(\begin{array}{lll}
(\sqrt{.05}, \sqrt{.08}) & (\sqrt{.30}, \sqrt{.12}) & (\sqrt{.24}, \sqrt{.12}) \\
(\sqrt{.30}, \sqrt{.12}) & (\sqrt{.14}, \sqrt{.06}) & (\sqrt{.20}, \sqrt{.06}) \\
(\sqrt{.24}, \sqrt{.12}) & (\sqrt{.20}, \sqrt{.06}) & (\sqrt{.40}, \sqrt{.03})
\end{array}\right)
$$

Here

$$
(A \$ B)^{T}=\left(\begin{array}{lll}
(\sqrt{.05}, \sqrt{.08}) & (\sqrt{.30}, \sqrt{.12}) & (\sqrt{.24}, \sqrt{.12}) \\
(\sqrt{.30}, \sqrt{.12}) & (\sqrt{.14}, \sqrt{.06}) & (\sqrt{.20}, \sqrt{.06}) \\
(\sqrt{.24}, \sqrt{.12}) & (\sqrt{.20}, \sqrt{.06}) & (\sqrt{.40}, \sqrt{.03})
\end{array}\right)
$$

This implies $(A \$ B)^{T}=A \$ B$ and hence $A \$ B$ is again a symmetric intuitionistic fuzzy soft matrix.

\section{Proof of $v i$}

Let A be a symmetric intuitionistic fuzzy soft matrix such that

$$
A=\left(\begin{array}{lll}
(.1, .2) & (.5, .4) & (.3, .6) \\
(.5, .4) & (.2, .3) & (.5, .1) \\
(.3, .6) & (.5, .1) & (.6, .2)
\end{array}\right)
$$

and

$$
A^{T}=\left[\begin{array}{lll}
(.1, .2) & (.5, .4) & (.5, .2) \\
(.5, .4) & (.2, .3) & (.3, .4) \\
(.3, .6) & (.5, .1) & (.6, .2)
\end{array}\right]
$$

Then $\mathrm{A}^{\mathrm{T}} \mathrm{A}$ is to be calculated.

Let $A^{T} A=\left[A_{i j}\right]$, where

$\mathrm{A}_{11}=[\max (.1, .5, .3), \min (.2, .4, .6)]$ $=(.5, .2)$

$\mathrm{A}_{12}=[\max (.1, .2, .3), \min (.4, .4, .6)]$ $=(.3, .4)$

$\mathrm{A}_{13}=[\max (.1, .5, .3), \min (.6, .4, .6)]$ $=(.5, .4)$

$\mathrm{A}_{21}=[\max (.1, .2, .3), \min (.4, .4, .6)]$ $=(.3, .4)$

$\mathrm{A}_{22}=[\max (.5, .2, .5), \min (.4, .3, .1)]$ $=(.5, .1)$ 
$\mathrm{A}_{23}=[\max (.3, .2, .5), \min (.6, .2, .2)]$ $=(.5, .2)$

$\mathrm{A}_{31}=[\max (.1, .5, .3), \min (.6, .4, .6)]$ $=(.5, .4)$

$\mathrm{A}_{32}=[\max (.3, .2, .5), \min (.6, .3, .2)]$ $=(.5, .2)$

$\mathrm{A}_{33}=[\max (.3, .5, .6), \min (.6, .1, .2)]$ $=(.6, .1)$

Thus

$$
A^{T} A=\left[\begin{array}{lll}
(.5, .2) & (.3, .4) & (.5, .4) \\
(.3, .4) & (.5, .1) & (.5, .2) \\
(.5, .4) & (.5, .2) & (.6, .1)
\end{array}\right]
$$

From the above result, it is clear that $\mathrm{A}^{\mathrm{T}} \mathrm{A}$ is also a symmetric intuitionistic fuzzy soft matrix.

Proof of vii

Let $\mathrm{A}$ and $\mathrm{B}$ as mentioned above are two symmetric intuitionistic soft fuzzy matrices. Then it is to be proved that $\mathrm{A}^{\mathrm{T}} \mathrm{BA}$ is again a symmetric intuitionistic fuzzy soft matrix.

Here let $\mathrm{A}^{\mathrm{T}} \mathrm{B}=\left[\mathrm{E}_{\mathrm{ij}}\right]$, where

$\mathrm{E}_{11}=(.5, .4), \mathrm{E}_{12}=(.5, .3), \mathrm{E}_{13}=(.4, .2), \mathrm{E}_{21}=(.5, .2)$, $\mathrm{E}_{22}=(.5, .3), \mathrm{E}_{23}=(.5, .1), \mathrm{E}_{31}=(.6, .2), \mathrm{E}_{32}=(.5, .2)$ and $\mathrm{E}_{33}=(.6, .2)$.

Hence

$$
A^{T} B=\left(\begin{array}{lll}
(.5, .4) & (.5, .3) & (.4, .2) \\
(.5, .2) & (.5, .3) & (.5, .1) \\
(.6, .2) & (.5, .2) & (.6, .2)
\end{array}\right)
$$

Again if $A^{T} B A=\left[G_{i j}\right]$, then

$\mathrm{G}_{11}=(.5, .4), \mathrm{G}_{12}=(.5, .2), \mathrm{G}_{13}=(.5, .2), \mathrm{G}_{21}=(.5, .2)$, $\mathrm{G}_{22}=(.5, .1), \mathrm{G}_{23}=(.5, .2), \mathrm{G}_{31}=(.5, .2), \mathrm{G}_{32}=(.5, .2)$ and $\mathrm{G}_{33}=(.6, .2)$.

Thus

$$
A^{T} B A=\left(\begin{array}{lll}
(.5, .4) & (.5, .2) & (.5, .2) \\
(.5, .2) & (.5, .1) & (.5, .2) \\
(.5, .2) & (.5, .2) & (.6, .2)
\end{array}\right)
$$

From the above, it is clear that $\mathrm{A}^{\mathrm{T}} \mathrm{BA}$ is again a symmetric intuitionistic fuzzy soft matrix.

\section{Proof of viii}

From above, we have

$$
A \cup B=\left(\begin{array}{lll}
(.5, .2) & (.6, .3) & (.8, .2) \\
(.6, .3) & (.7, .2) & (.5, .1) \\
(.8, .2) & (.5, .1) & (.8, .1)
\end{array}\right)
$$

Then it can be obtained that

$$
\begin{aligned}
& (A \cup B) \$ C \\
& =\left(\begin{array}{lll}
(\sqrt{.15}, \sqrt{.08}) & (\sqrt{.30}, \sqrt{.12}) & (\sqrt{.48}, \sqrt{.02}) \\
(\sqrt{.30}, \sqrt{.12}) & (\sqrt{.42}, \sqrt{.06}) & (\sqrt{.20}, \sqrt{.06}) \\
(\sqrt{.48}, \sqrt{.02}) & (\sqrt{.20}, \sqrt{.06}) & (\sqrt{.56}, \sqrt{.02})
\end{array}\right)
\end{aligned}
$$

Thus it is seen that if $\mathrm{A}, \mathrm{B}$ and $\mathrm{C}$ be three symmetric intuitionistic fuzzy soft matrices then $(A \cup B) \$ C$ is again a symmetric intuitionistic fuzzy soft matix.

\section{Proof of ix}

Proceeding as above, it can be shown that if A, B and $\mathrm{C}$ be two symmetric intuitionistic fuzzy matrix, then $(A \cap B) \$ C$ is again a symmetric intuitionistic fuzzy soft matrix.

\section{Proof of $x$}

Let $A=\left(\mu_{i j}^{A}, v_{i j}^{A}\right), B=\left(\mu_{i j}^{B}, v_{i j}^{B}\right)$ and $C=\left(\mu_{i j}^{C}, v_{i j}^{C}\right)$ be three symmetric intuitionistic soft fuzzy matrices. Then

$$
A @ B=\left(\frac{\mu_{i j}^{A}+\mu_{i j}^{B}}{2}, \frac{v_{i j}^{A}+v_{i j}^{B}}{2}\right)
$$

and

$$
\begin{aligned}
& (A @ B) \$ C \\
& =\left(\sqrt{\left(\frac{\left(\mu_{i j}^{A}+\mu_{i j}^{B}\right) \mu_{i j}^{C}}{2}\right)}, \sqrt{\left(\frac{\left(v_{i j}^{A}+v_{i j}^{B}\right) v_{i j}^{C}}{2}\right)}\right)
\end{aligned}
$$

Since A, B and C are symmetric intuitionistic fuzzy soft matrices then

$$
\begin{aligned}
& \left(\sqrt{\left(\frac{\left(\mu_{i j}^{A}+\mu_{i j}^{B}\right) \mu_{i j}^{C}}{2}\right)}, \sqrt{\left(\frac{\left(v_{i j}^{A}+v_{i j}^{B}\right) v_{i j}^{C}}{2}\right)}\right) \\
& =\left(\sqrt{\left(\frac{\left(\mu_{j i}^{A}+\mu_{j i}^{B}\right) \mu_{j i}^{C}}{2}\right)}, \sqrt{\left(\frac{\left(v_{j i}^{A}+v_{j i}^{B}\right) v_{j i}^{C}}{2}\right)}\right)
\end{aligned}
$$

This shows that $(A @ B) \$ C$ is again a symmetric intuitionistic fuzzy soft matrix.

\section{Numerical Example}

We have

$$
A @ B=\left(\begin{array}{ccc}
(.3, .3) & (.55, .35) & (.55, .4) \\
(.55, .35) & (.45, .25) & (.45, .35) \\
(.55, .4) & (.45, .35) & (.65, .2))
\end{array}\right)
$$


and then it can be obtained that

$$
\begin{aligned}
& (A @ B) \$ C \\
& =\left(\begin{array}{ccc}
(\sqrt{.09}, \sqrt{.12}) & (\sqrt{.275}, \sqrt{.140}) & (\sqrt{.330}, \sqrt{.04}) \\
(\sqrt{.275}, \sqrt{.140}) & (\sqrt{.270}, \sqrt{.025}) & (\sqrt{.180}, \sqrt{.210}) \\
(\sqrt{.330}, \sqrt{.04}) & (\sqrt{.180}, \sqrt{.210}) & (\sqrt{.455}, \sqrt{.04})
\end{array}\right)
\end{aligned}
$$

This proves the property $(x)$.

\section{CONCLUSION}

Here the main intention was to study the transpose of intuitionistic fuzzy matrices and symmetric intuitionistic fuzzy soft matrices. Some of the properties of transpose of intuitionistic fuzzy soft matrices have been discus sed and verified with numerical examples. Further, symmetric intuitionistic soft fuzzy matrices are defined and some properties associated with symmetric intuitionistic fuzzy matrices are discussed with numerical examples. It is seen that some properties of transpose of intuitionistic fuzzy soft matrices and also those of symmetric intuitionistic fuzzy matrices coincides with those of classical matrices and fuzzy matrices. In future, the main concentration will be in the direction of considering all the other properties of classical matrices in terms of intuitionistic fuzzy soft matrices.

\section{ACKNOWLEDGEMENT}

The author is grateful to all the referees for their valuable suggestions in the preparation of the article.

\section{REFERENCES}

[1] Zadeh, L.A., Fuzzy Sets, Information and Control, 1965, $8,338-353$

[2] Atanassov, K.T., "Intuitionistic Fuzzy Sets”, Fuzzy Sets and Sy stems, 1986, 20(1), 87-96.

[3] Szmidt, E., Kacprzyk, J., "Intuitionistic fuzzy sets in some medical applications", Note on IFS, 2001, 7 (4), 58-64.

[4] Szmidt, E., Kacprzyk, J., "Medical diagnostic reasoning using a similarity measure for intuitionistic fuzzy sets", Note on IFS, 2004, 10 (4), 61-69.

[5] Atanassov, K.T., "Intuitionistic fuzzy sets: theory and application", Springer, 1999

[6] Atanassov, K.T., "On Intuitionistic fuzzy sets", Springer 2012.

[7] Molodtsov, D.A.,"Soft Set Theory First Results" Computers and Mathematics with Applications, 1999, 37, 19-31.

[8] Maji, P.K. ,Biswas, R., and Roy, A.R., "Soft Set Theory", Computer and Mathematics with Applications, 2003, 45, 555-562.

[9] Maji, P.K. ,Biswas, R., and Roy, A.R, "Intuitionistic Fuzzy Soft Sets”, Journal of Fuzzy Mathematics, 2001, 9 , 677-692.

[10] Yong Yang and Chenli, Ji, "Fuzzy Soft Matrices and their Applications", Part 1, LNAI, 2011, 618-627, 7002.

[11] Broumi, S., Smarandache, F., Dhar, M., and Majumbar,P., "New Results of Intuitionistic Fuzzy Soft Sets",International Journal of Enjgineering and Electronic Business, 2014, 2, 47-52
[12] Chetia, B., Das, P. K. , "On Fuzzy Soft Matrix Theory”, Journal of Assam Academy of Mathematics, 2010,2, 71-83

[13] Verma, R., Dev Sharma, B., "Some new Results on Intuitionistic Fuzzy Sets", Proceeding of the Jangjeon Mathematical Society, 2013, 16(1), 101-114

[14] Rajarajeswari, P., Dhanalakshmi, P., Intuitionistic fuzzy soft matrix theory and its application in decision making, International Journal of Engineering Research \& Technology, 2013, 2 (4) .

[15] Dhar, M., Study of some properties of intuitionistic fuzzy soft matrix, International Journal of Information Engineering and Electronic Business, 2016, 8(2), 37-45.

\section{Authors' Profiles}

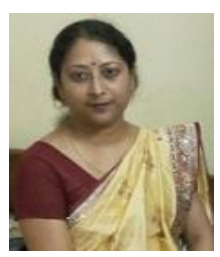

Mamoni Dhar is an Assiatant Professor in the Department of Mathematics, Science College, Kokrajhar. She obtained her Ph.D from Gauhati University, Assam, India. Her research interest is in the theory of fuzzy sets.

How to cite this paper: Mamoni Dhar, "Some Results of Intuitionistic Fuzzy Soft Matrix", International Journal of Intelligent Systems and Applications (IJISA), Vol.8, No.8, pp. 48-57, 2016. DOI: 10.5815 /ijisa.2016.08.06 